\title{
Re-visiting the functional Relevance of the highly conserved Serine 40 Residue within HIV-1 p6 ${ }^{\mathrm{Gag}}$
}

\author{
Benjamin Radestock, Robin Burk, Barbara Müller and Hans-Georg Kräusslich*
}

\begin{abstract}
Background: HIV-1 formation is driven by the viral structural polyprotein Gag, which assembles at the plasma membrane into a hexagonal lattice. The C-terminal p6 ${ }^{\text {Gag }}$ domain harbors short peptide motifs, called late domains, which recruit the cellular endosomal sorting complex required for transport and promote HIV-1 abscission from the plasma membrane. Similar to late domain containing proteins of other viruses, HIV-1 p6 is phosphorylated at multiple residues, including a highly conserved serine at position 40 . Previously published studies showed that an S4OF exchange in $\mathrm{p6}^{\mathrm{Gag}}$ severely affected virus infectivity, while we had reported that mutation of all phosphorylatable residues in $\mathrm{p}^{\mathrm{Gag}}$ had only minor effects.

Findings: We introduced mutations into $p 6^{\text {Gag }}$ without affecting the overlapping pol reading frame by using an HIV-1 derivative where gag and pol are genetically uncoupled. HIV-1 derivatives with a conservative S40N or a non-conservative S40F exchange were produced. The S40F substitution severely affected virus maturation and infectivity as reported before, while the $S 40 \mathrm{~N}$ exchange caused no functional defects and the variant was fully infectious in T-cell lines and primary T-cells.

Conclusions: An HIV-1 variant carrying a conservative $\$ 40 \mathrm{~N}$ exchange in $\mathrm{p}^{\text {Gag }}$ is fully functional in tissue culture demonstrating that neither $\mathrm{S} 40$ nor its phosphorylation are required for HIV-1 release and maturation. The phenotype of the S4OF mutation appears to be caused by the bulky hydrophobic residue introduced into a flexible region.
\end{abstract}

Keywords: Human immunodeficiency virus, Gag, p6, Phosphorylation, Release, Serine 40

\section{Findings}

HIV-1 assembly is driven by the viral Gag polyprotein. Gag is necessary and sufficient for particle formation, and is composed of four functional subunits. The $\mathrm{N}$-terminal MA domain targets Gag to the plasma membrane, where $\sim 2,500$ Gag molecules form the curved, hexagonal immature lattice of HIV-1, which is stabilized by intermolecular CA domain interactions. The NC domain is responsible for packaging viral genomic RNA [1]. Crucial motifs for particle release lie within the 52 amino acids long C-terminal $\mathrm{p}^{\mathrm{Gag}}$ domain. This domain harbors so-called late domains, which recruit the cellular endosomal sorting complex required for transport (ESCRT) promoting abscission of progeny particles from the host cell. Other motifs in $\mathrm{p} 6^{\mathrm{Gag}}$ mediate incorporation of the viral accessory protein Vpr into HIV-1 particles [2-4]. During maturation,

\footnotetext{
* Correspondence: Hans-Georg.Kraeusslich@med.uni-heidelberg.de Department of Infectious Diseases, Virology, University Hospital Heidelberg, Im Neuenheimer Feld 324, D-69120 Heidelberg, Germany
}

immature HIV-1 gains infectivity following proteolytic cleavage of Gag into its functional domains by the viral protease [1].

$\mathrm{p} 6^{\mathrm{Gag}}$ has been shown to be the predominant phosphoprotein in HIV-1 particles [5]. It is phosphorylated at several positions, including the highly conserved residue $\mathrm{S} 40$ [5-8]. S40 phosphorylation has been detected in infected cells and viral particles [7], and this residue can be phosphorylated by atypical protein kinase $\mathrm{C}(\mathrm{aPKC})$ in vitro [6].

To determine the role of $\mathrm{p} 6$ phosphorylation for HIV-1 replication, we had recently performed a comprehensive mutational analysis of p6 [7]. The use of an HIV-1 $1_{\text {NL4-3 }}$ based proviral plasmid with genetically uncoupled gag and pol open reading frames (ORFs) (pNL4-3 $3_{\text {unc }}$ ) allowed us to freely introduce mutations in the $\mathrm{p} 6^{\text {gag }}$ encoding region without affecting the pol ORF. In this context, we changed all phosphorylatable residues (i.e., Ser, Thr, Tyr) within $\mathrm{p}^{\mathrm{Gag}}$ with exception of the essential threonine in the PTAP late domain motif. The resulting virus, NL4- $3_{\text {unc }} \mathrm{FL}$ 
exhibited no significant difference in replication capacity compared to wild-type. This result led us to conclude that p6 phosphorylation is dispensable for viral morphogenesis and replication in cell culture.

In contrast, previous studies had reported that an S40F change in $\mathrm{p}^{\mathrm{Gag}}$ impaired proteolytic maturation of Gag, reduced viral infectivity and delayed replication in T-cell lines $[9,10]$. Furthermore, enhanced membrane binding affinity of a synthetic p6 C-terminal fragment was observed in vitro upon substitution of Ser40 by Phe or upon adding a phosphate group to this residue [11]. The S40F exchange was furthermore shown to result in an enhanced interaction of Gag with the ESCRTassociated protein Alix [9]. Taken together, these studies suggested an important role of S40 in Gag assembly [9], viral maturation [10], $\mathrm{Vpr}$ incorporation [6], and p6 membrane binding [11], in apparent contradiction to our observation that an HIV-1 derivative carrying mutations at 12 positions within p6, including S40, was fully functional in cell culture [7].

A major difference between our work [7] and the studies reported by others [6,9-11] was that the latter employed a chemically drastic Ser to Phe exchange in order to maintain the amino acid sequence of the overlapping pol ORF, whereas the gag-pol uncoupling strategy allowed us to select the most conservative substitution, Ser to Asn. In order to resolve the apparent discrepancies between our study and data published by others, we performed a direct side-by-side comparison of viruses carrying either an Asn or a Phe residue at position 40 of $\mathrm{p} 6^{\mathrm{Gag}}$.

The analysis included the previously described proviral plasmids pNL4- $3_{\text {unc }}$ with uncoupled wild-type $g a g$ and $p o l$ and pNL4- $3_{\text {unc }} \mathrm{FL}$, in which all phosphorylatable residues in $\mathrm{p}^{\mathrm{Gag}}$ except for $\mathrm{T} 8$, which is required for L-domain function [12], had been changed to chemically similar, but not phosphorylatable residues [7]. A derivative of pNL4- $3_{\text {unc }} \mathrm{FL}$ in which the substitution at position 40 of $\mathrm{p}^{\mathrm{Gag}}$ was reversed to the wild-type Ser-codon while retaining all other substitutions (pNL4- $\left.3_{\text {unc }} \mathrm{FL}-\mathrm{N} 40 \mathrm{~S}\right)$ was also included (Figure 1A). Mutant viruses were produced by transfection of HEK 293 T cells [13] using calcium phosphate and tested for efficiency of particle formation, Gag processing, $\mathrm{Vpr}$ incorporation, and infectivity. Controls included a release deficient late domain-defective variant (NL4-3 late(-), [14]), a derivative carrying alanine substitutions in the FRFG motif of $\mathrm{p}^{\mathrm{Gag}}$ and impaired in Vpr incorporation (NL4-3 $\operatorname{Vpr}(-)$, [3]), and a derivative which does not express $\mathrm{Vpr}(\mathrm{NL} 4-3 \Delta \mathrm{Vpr})$.

For assessment of virus release, culture media were harvested $30 \mathrm{~h}$ post transfection (p.t.), cleared by brief centrifugation followed by ultracentrifugation through a $20 \%(\mathrm{w} / \mathrm{w})$ sucrose cushion to pellet virus particles. Samples of cell and particle lysates were separated by SDS-PAGE and proteins were transferred to a PVDF membrane. HIV-1 CA-containing proteins were detected by quantitative immunoblotting using polyclonal sheep antiserum against recombinant CA (Figure 1B), and HIV1 particle release was quantified by determining the ratio of the amount of pelletable extracellular CA-containing proteins over the total amount of CA-containing proteins (Figure 1C). As expected, NL4-3 late(-) showed strongly reduced particle release compared to wild-type, accompanied by a characteristic increase in the proportion of processing intermediates, in particular CA-SP1. Variants NL4-3 $3_{\text {unc }}$ FL, FL-N40S, and S40N displayed wild-type Gag processing and particle release. Increased amounts of the CA-SP1 processing intermediate were observed in case of the S40F variant, consistent with previous reports $[9,10]$, and particle release was also slightly higher in this case (Figure 1B,C).

Kudoh and coworkers reported that (i) an S40A substitution in $\mathrm{p}^{\mathrm{Gag}}$ abolished incorporation of exogenously expressed Vpr into virus-like Gag particles (VLPs) and (ii) a PKC inhibitor, presumed to prevent phosphorylation of S40, impaired HIV-1 replication in primary macrophages [6]. These authors proposed that S40 phosphorylation may be required for $\mathrm{Vpr}$ incorporation into HIV-1 particles, and may thus be functionally relevant for replication in non-dividing cells, where the accessory protein Vpr is required [16]. In our previous analyses, we had not observed a block of Vpr incorporation in the case of pNL4$3_{\text {unc }} \mathrm{FL}$, where all phosphorylatable residues including $\mathrm{S} 40$ had been mutated [7]. This was confirmed in the present study, using a polyclonal rabbit antiserum raised against synthetic HIV-1 Vpr. Our analysis further revealed that neither the S40F nor the S40N exchange altered Vpr incorporation into HIV-1 (Figure 2). We conclude that the Vpr incorporation block reported before [6] is either a specific property of the S40A variant, or, more likely, represents a feature of the $\mathrm{Gag} / \mathrm{Vpr}$ overexpression system used in the prior study that is not observed in the native viral context.

Single round infectivity of wild-type and mutant virus preparations was assessed by titration on HeLa TZM-bl indicator cells [17] (Figure 3A). Viral titers were determined by endpoint titration on C8166 T-cells [18] (Figure 3B). Neither single-round infectivity nor viral titer of the variants HIV- $1_{\mathrm{NL} 4-3 \text { unc }} \mathrm{FL}$, FL-N40S, or S40N, respectively, differed significantly from that of wild-type HIV- $1_{\text {NL4-3unc }}$ (Figure 3A,B). In line with published results [9,10], infectivity was severely decreased for the S40F variant with a similar reduction as observed for the release deficient late(-) control.

Finally, we investigated the replication kinetics of the mutant HIV-1 panel in primary human peripheral blood mononuclear cells (PBMC) (Figure 4). PBMC obtained from three healthy blood donors were mixed, activated for 3 days with $2 \mu \mathrm{g} / \mathrm{ml}$ PHA (Sigma) and $10 \mu \mathrm{g} / \mathrm{ml} \mathrm{IL-2}$ 


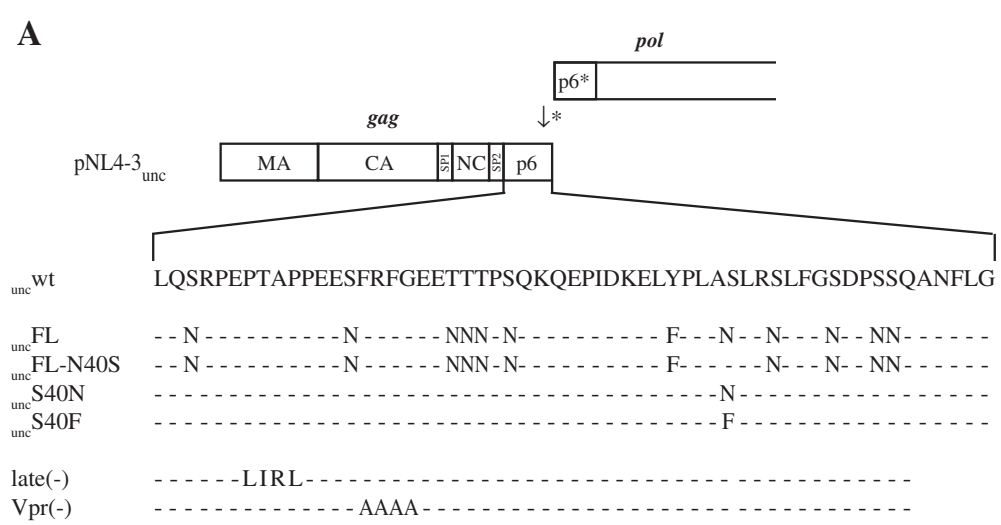

\section{B}

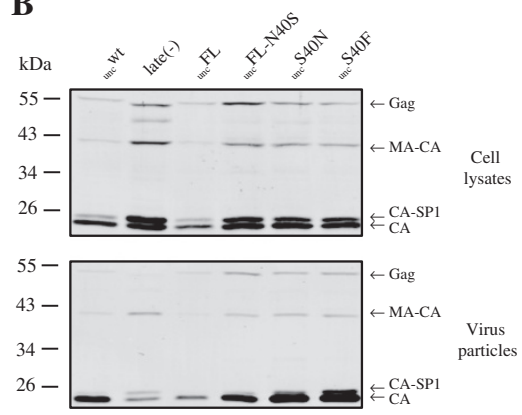

C

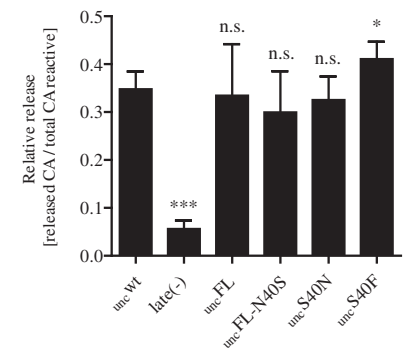

Figure 1 HIV-1 p $^{\text {Gag }}$ variants and their effect on Gag processing and viral release. (A) Scheme of the gag and pol ORFs in the HIV-1 NL4-3unc genome [15]. The arrow with an asterisk indicates the frameshift signal at the $3^{\prime}$ end of gag. Amino acid sequences of the altered p6 sequences of the NL4-3 unc variants are shown below; mutated residues are indicated. The NL4-3 late(-) and Vpr(-) variants were constructed in the wild-type pNL4-3 proviral plasmid with overlapping gag and pol reading frames. (B) Gag processing and particle release efficiency. Virus particles were prepared by ultracentrifugation from the culture media of HEK 293 T cells transfected with the indicated proviral plasmids. Cell lysates (top panel) and virus particles (bottom panel) were analyzed for Gag-derived products by quantitative immunoblot (LiCor) using antiserum against HIV-1 CA. Positions of molecular mass standards are shown to the left, Gag-derived processing products are indicated to the right. (C) Quantitative immunoblotting of cell lysates and particles as shown in (B) was used to calculate the amount of released CA-containing proteins by quantification of band intensities using the LiCor Odyssey 3.0 software. Relative release was calculated by dividing the sum of CA-reactive band intensities in the particle fraction by total CA-reactive band intensities in cell lysates and particles. Mean values and SD from three independent experiments performed in duplicates are shown. $p$-values were calculated using an unpaired student's t-test (n.s. $>0.05,{ }^{*}<0.05,{ }^{* *}<0.001$ ).
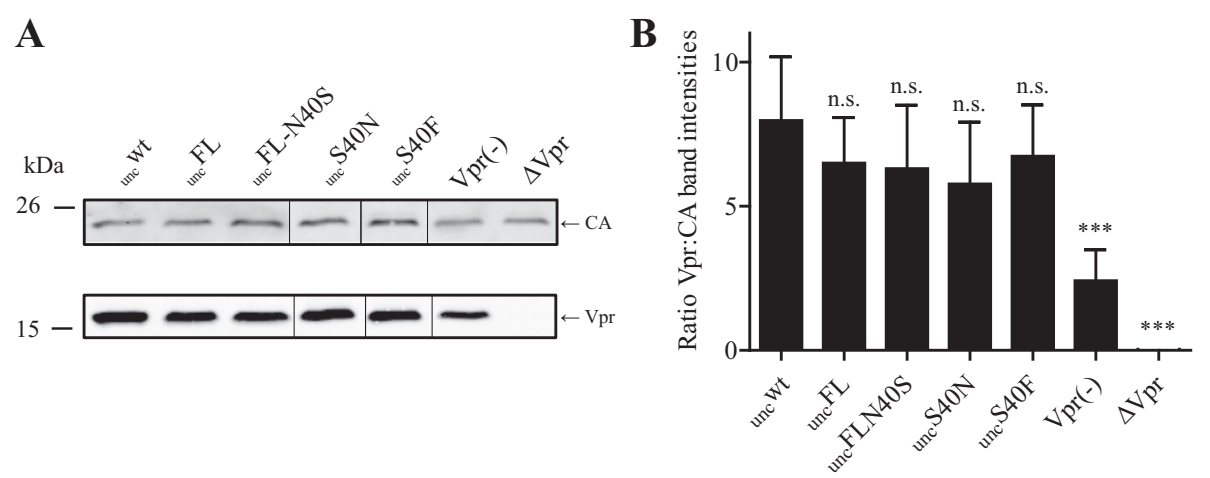

Figure 2 Vpr incorporation into HIV-1 particles. (A) Immunoblot analysis of Vpr incorporation. Virions were collected from culture media of HEK 293 T cells transfected with the respective proviral plasmids by ultracentrifugation through a sucrose cushion. NL4-3 Vpr(-) (generated by transferring a Spel - Agel fragment from pNL43_E-_R-_Luc3/p6:M1A ([3]) into pNL4-3) and NL4-3 $\Delta$ Vpr were used as negative controls. Samples were separated by SDS-PAGE, and viral particles were analyzed by quantitative immunoblot (LiCor) using antisera against recombinant HIV-1 CA (top) and Vpr (bottom). The positions of molecular mass standards are shown on the left and proteins are indicated on the right. (B) Quantification of Vpr incorporation. The ratio of Vpr to CA represents relative signal intensities from quantitative immunoblots, as shown in panel A. Mean values and SD from three independent experiments performed in duplicates are shown. $p$-values were calculated using an unpaired student's $t$-test (n.s. $>0.05,{ }^{* * *}<0.001$ ). 

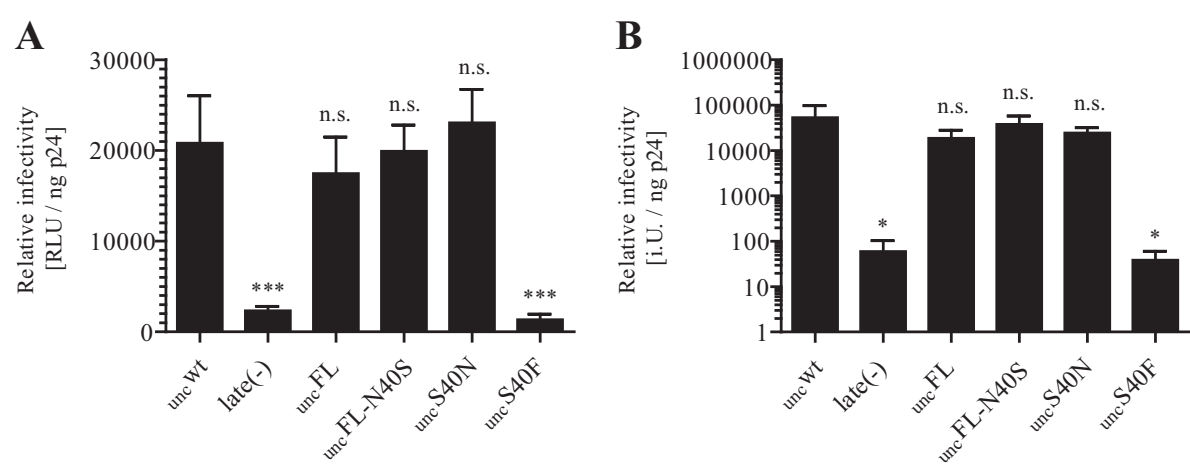

Figure 3 Effect of p6 S40 substitutions on viral infectivity. Viral particles were harvested from cell culture media of HEK 293 T cells transfected with proviral plasmids and tested for infectivity. Single round infectivity (A) was assessed by titration of viral particles on TZM-bl cells as described in the main text. Relative infectious titers on C8166 T-cells (B) were determined by endpoint titration. Values were normalized to p24 amounts determined by ELISA. Graphs show mean values and SD of one experiment using viral particles from six independent preparations. p-values in (A) and (B) were calculated using an unpaired student's t-test (n.s. $>0.05,{ }^{*}<0.05,{ }^{* * *}<0.001$ ).

(Biomol), and infected with virus obtained from transfected HEK 293 T cells. Virus replication was monitored over two weeks by collecting culture supernatant every second day and quantifying p24 release by ELISA. Replication of the late(-) (Figure 4A) and S40F variants (Figure 4B) was completely abolished in PBMC, consistent with their severe replication defect in established cell lines. The replication defect of the S40F mutant in PBMCs was even more severe than in a previous report analyzing replication in primary human lymphocyte aggregate cultures, where residual and delayed replication had been observed [10]. Most importantly, however, replication kinetics of the S40N, FL, and FL-N40S variants in PBMC were indistinguishable from wild-type HIV- $1_{\mathrm{NL} 4-3 \text { unc }}$ (Figure $4 \mathrm{~A}, \mathrm{~B}$ ), demonstrating that the serine residue itself and phosphorylation at this site are both dispensable for HIV-1 replication in primary T-cells.
In summary, our analyses confirm previous reports that an S40F substitution in $6^{\mathrm{Gag}}$ of HIV-1 impairs Gag processing at the CA-SP1 site and severely affects or abolishes $\mathrm{HIV}-1$ replication in cell lines and primary cells $[9,10]$. This effect is not due to either a requirement for the conserved serine residue at this position or for phosphorylation of S40. The conservative substitution of S40 by a chemically similar, but not phosphorylatable Asn residue had no effect on Gag processing or viral infectivity in cell lines or primary T-cells compared to wild-type HIV-1. Thus, the previously described replication defect of the S40F variant appears to be due to the replacement of the small, hydrophilic serine by a bulky hydrophobic phenylalanine residue, rather than indicating a requirement for S40. We did not analyze Gag membrane-binding properties, which had been reported to be affected by the S40F substitution or by introducing
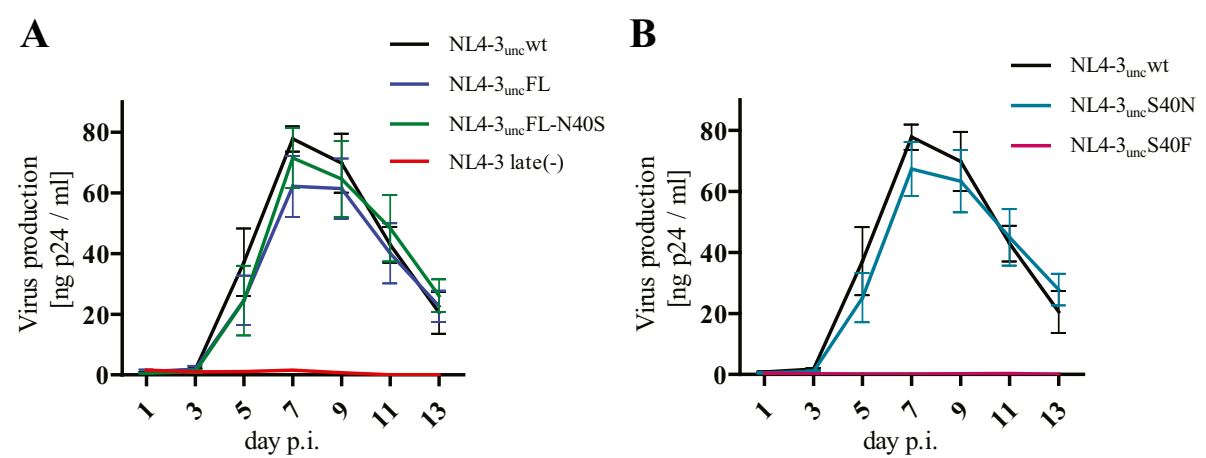

Figure 4 Replication kinetics of HIV-1 variants in PBMC. PBMC were isolated using buffy coats from healthy blood donors and stimulated for three days using PHA and IL-2. Subsequently, cells from three individual donors were mixed, and virus harvested from cell culture media of HEK 293 T cells transfected with the respective proviral plasmids was added in triplicates $\left(0.1 \mathrm{ng} \mathrm{p} 24 / 3 \times 10^{5} \mathrm{cells}\right)$. Samples of cell culture media were collected at the indicated time points and virus production was measured by p24 ELISA. Results from one infection experiment using six

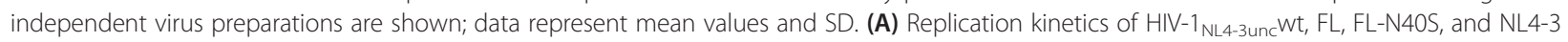

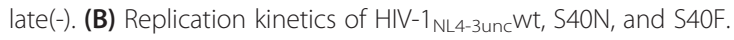


a phosphomimick in this position [11]. However, wildtype release, polyprotein processing and infectivity of all variants studied here except for S40F suggest that neither S40 nor its phosphorylation is needed for fully functional membrane binding of Gag. Furthermore, we did not observe a block in $\mathrm{Vpr}$ incorporation upon mutation of S40, which had been reported for an S40A variant in a previous study and had been proposed to result in impaired replication in primary macrophages [6]. While we cannot exclude that $\mathrm{S} 40$ in $\mathrm{p}^{\mathrm{Gag}}$ and/or its phosphorylation may be relevant in a different cell context (e.g., macrophages), we conclude that the phenotypes reported in previously published studies [6,9-11] were most likely caused by the specific mutation introduced and do not reflect the functional importance of Ser40 or its phosphorylation.

\section{Competing interests}

The authors declare that they have no competing interests.

\section{Authors' contributions}

$\mathrm{BR}, \mathrm{BM}$, and HGK designed experiments. BR and RB performed experiments and analyzed data. All authors contributed to data interpretation and writing of the manuscript.

\section{Acknowledgements}

We thank Bärbel Glass for technical assistance. The plasmid pNL43_E-_R-_Luc3/ p6:M1A, which was used to produce pNL4-3 $\operatorname{Vpr}(-)$, was kindly provided by Nathaniel Landau (New York). This work was supported in part by the Deutsche Forschungsgemeinschaft through the collaborative research grant SFB638 (project A9). HGK and BM are investigators of the excellence cluster CellNetworks (EXC81). RB is supported by the Hartmut Hoffman-Berling International Graduate School of Molecular and Cellular Biology.

Received: 23 September 2014 Accepted: 17 November 2014 Published online: 19 December 2014

\section{References}

1. Sundquist WI, Krausslich HG: HIV-1 Assembly, Budding, and Maturation. Cold Spring Harb Perspect Med 2012, 2:a006924.

2. Kondo E, Gottlinger HG: A conserved LXXLF sequence is the major determinant in p6gag required for the incorporation of human immunodeficiency virus type 1 Vpr. J Virol 1996, 70:159-164.

3. Sunseri N, O'Brien M, Bhardwaj N, Landau NR: Human immunodeficiency virus type 1 modified to package Simian immunodeficiency virus Vpx efficiently infects macrophages and dendritic cells. J Virol 2011, 85:6263-6274.

4. Paxton W, Connor Rl, Landau NR: Incorporation of Vpr into human immunodeficiency virus type 1 virions: requirement for the p6 region of gag and mutational analysis. J Virol 1993, 67:7229-7237.

5. Muller B, Patschinsky T, Krausslich HG: The Late-Domain-Containing Protein p6 Is the Predominant Phosphoprotein of Human Immunodeficiency Virus Type 1 Particles. J Virol 2002, 76:1015-1024.

6. Kudoh A, Takahama S, Sawasaki T, Ode H, Yokoyama M, Okayama A Ishikawa A, Miyakawa K, Matsunaga S, Kimura H, Suqiura W, Sato H, Hirano H, Ohno S, Yamamoto N, Ryo A: The phosphorylation of HIV-1 Gag by atypical protein kinase $\mathrm{C}$ facilitates viral infectivity by promoting $\mathrm{Vpr}$ incorporation into virions. Retrovirology 2014, 11:9.

7. Radestock B, Morales I, Rahman SA, Radau S, Glass B, Zahedi RP, Muller B, Krausslich HG: Comprehensive mutational analysis reveals p6Gag phosphorylation to be dispensable for HIV-1 morphogenesis and replication. J Virol 2013, 87:724-734.

8. Hemonnot B, Cartier C, Gay B, Rebuffat S, Bardy M, Devaux C, Boyer V, Briant $L$ : The host cell MAP kinase ERK-2 regulates viral assembly and release by phosphorylating the p6gag protein of HIV-1. J Biol Chem 2004, 279:32426-32434.
9. Watanabe SM, Chen MH, Khan M, Ehrlich L, Kemal KS, Weiser B, Shi B, Chen C, Powell M, Anastos K, Burger H, Carter CA: The S40 residue in HIV-1 Gag p6 impacts local and distal budding determinants, revealing additional late domain activities. Retrovirology 2013, 10:143.

10. Votteler J, Neumann L, Hahn S, Hahn F, Rauch P, Schmidt K, Studtrucker N, Solbak SM, Fossen T, Henklein P, Ott DE, Holland G, Bannert N, Schubert U: Highly conserved serine residue 40 in HIV-1 p6 regulates capsid processing and virus core assembly. Retrovirology 2011, 8:11.

11. Solbak SM, Reksten TR, Hahn F, Wray V, Henklein P, Henklein P, Halskau O, Schubert U, Fossen T: HIV-1 p6 - a structured to flexible multifunctional membrane-interacting protein. Biochim Biophys Acta 1828, 2013:816-823.

12. Gottlinger HG, Dorfman T, Sodroski JG, Haseltine WA: Effect of mutations affecting the p6 gag protein on human immunodeficiency virus particle release. Proc Natl Acad Sci U S A 1991, 88:3195-3199.

13. Sena-Esteves M, Saeki Y, Camp SM, Chiocca EA, Breakefield XO: Single-step conversion of cells to retrovirus vector producers with herpes simplex virus-Epstein-Barr virus hybrid amplicons. J Virol 1999, 73:10426-10439.

14. Huang M, Orenstein JM, Martin MA, Freed EO: p6Gag is required for particle production from full-length human immunodeficiency virus type 1 molecular clones expressing protease. J Virol 1995, 69:6810-6818.

15. Adachi A, Gendelman HE, Koenig S, Folks T, Willey R, Rabson A, Martin MA: Production of acquired immunodeficiency syndrome-associated retrovirus in human and nonhuman cells transfected with an infectious molecular clone. J Virol 1986, 59:284-291.

16. Connor Rl, Chen BK, Choe S, Landau NR: Vpr is required for efficient replication of human immunodeficiency virus type-1 in mononuclear phagocytes. Virology 1995, 206:935-944.

17. Wei X, Decker JM, Liu H, Zhang Z, Arani RB, Kilby JM, Saag MS, Wu X, Shaw GM, Kappes JC: Emergence of resistant human immunodeficiency virus type 1 in patients receiving fusion inhibitor (T-20) monotherapy. Antimicrob Agents Chemother 2002, 46:1896-1905.

18. Salahuddin SZ, Markham PD, Wong-Staal F, Franchini G, Kalyanaraman VS, Gallo RC: Restricted expression of human T-cell leukemia-lymphoma virus (HTLV) in transformed human umbilical cord blood lymphocytes. Virology 1983, 129:51-64.

\section{Submit your next manuscript to BioMed Central and take full advantage of:}

- Convenient online submission

- Thorough peer review

- No space constraints or color figure charges

- Immediate publication on acceptance

- Inclusion in PubMed, CAS, Scopus and Google Scholar

- Research which is freely available for redistribution 